\title{
ON THE COMPLEXITY AND EFFICIENCY OF A NEW KEY EXCHANGE SYSTEM
}

\author{
Johamnes A. Buchmann' Stephan Düllmann' \\ Hugh C. Williams') \\ 1) FB 10 Informatik \\ Universität des Saarlandes \\ D-6600 Saarbrïcken \\ WEST GERMANY \\ 2) Department of Computer Science \\ University of Manitoba \\ Winnipeg, Manitoba \\ CANADA R3T 2N2
}

\section{ABSTRACT}

In [2] Buchmann and Williams presented a new public key exchange system based on imaginary quadratic fields. While in that paper the system was described theoretically and its security was discussed in some detail nothing much was said about the practical implementation.

In this paper we discuss the practical aspects of the new system, its efficiency and implementation. In particular we study the crucial point of the method: ideal reduction. We suggest a refinement of the well known reduction method which has been implemented on a computer. We present extensive running time statistics and a detailed complexity analysis of the methods involved.

The implementation of the reduction procedure on chips is subject of future research.

\section{THE DIFFIE-HELLMAN SCHEME}

In their paper "New Directions in Cryptography" [3] Diffe and Hellman introduced in 1976 the idea of public key exchange. By this method it is 
possible to communicate a secret ley for some cryptosystem over a public insecure chamel. We briefly review the iclea of Diffie ancl Hellman.

Suppose that Alice $(\mathcal{A})$ and Bob (B) wish to secretly exchange a key.

(1) $\mathcal{A}$ chooses a finite group $C_{r}^{\prime}$ and - at random - an element. $\lambda \in C^{\prime}$. Both $G$ and $\lambda$ are sent to $B$ over the public channel.

(2) Both $\mathcal{A}$ and $B$ now select - at random - an integer $a$ and $b$, respectively. These integers are kept secret.

(3) $\mathcal{A}$ computes $\alpha=\lambda^{a}$ and transmits the result to $\mathcal{B}$. In the same way $\mathcal{B}$ computes $\beta=\lambda^{b}$ and sends it to $\mathcal{A}$. (Note that the group elements $\alpha$ and $\beta$ are public.)

(4) $\mathcal{A}$ computes $\gamma=\beta^{a}, \mathcal{B}$ computes $\gamma^{\prime}=\alpha^{b}$.

Because $\gamma=\beta^{a}=\left(\lambda^{b}\right)^{a}=\left(\lambda^{a}\right)^{b}=\alpha^{b}=\gamma^{\prime}$ both $\mathcal{A}$ and $B$ are in possession of the same key $\gamma$ without this key having been sent over the public channel.

In order to be able to apply this algorithm in practice it is necessary to have an efficient multiplication routine in $G$ i.e., if all the elements are represented by numbers in $\{0,1, \ldots,|G|-1\}$ the representation of the product of two elements of $G$ should be computable in time polynomial in $\log |G|$.

Then, using the method of binary shifting (see [6, p. $441 \mathrm{ff}$.$] ) pow-$ ers of group elements can be computed very efficiently even for large exponents $d$, namely in $O(\log d)$ elementary operations in $G$.

The scheme is secure if the key cannot be guessed easiliy and cannot be determined easily from the public information.

To avoid the key beeing guessed easily it is necessary to choose a group $G$ of very high order, to pick a starting element $\lambda$ of high order (close to $|G|$ ) and to select large exponents $a$ and $b$.

To make sure that the choice of $G$ is adequate one has to analyse its arithmetic properties carefully. A neccessary condition is that the determination of discrete logarithms in $G$ is difficult. Note, however, that this condition is not sufficient for the security of the system because the determination of $\lambda^{a b}$ from $\lambda, \lambda^{a}$ and $\lambda^{b}$ might be easier than the calculation of $a$ and $b$ from $\lambda, \lambda^{a}$ and $\lambda^{b}$.

So far the following groups have been suggested: 
- The group $G=G L_{n}(\mathscr{Z} / p \mathscr{Z})$ of invertible $n \times n$-matrices over the finite field $(\mathscr{Z} / p \mathscr{Z})$. ([14])

- The group of points on an elliptic curve over a finite field. ([11])

- Several groups associated with higher dimensional varieties. ([7])

- The group $G=(\mathscr{Z} / n \mathscr{Z})$ where $n$ is the product of two large primes. ([10])

- The class group of an imaginary quadratic field. ([2])

In this paper we continue the discussion of the case where $G$ is the class group of an imaginary quadratic field.

\section{THE CLASS GROUP OF AN IMAGINARY QUADRA- TIC FIELD}

First we summarize the main facts concerning imaginary quadratic fields. All these facts are well known and therefore will be given without proof. Proofs of the statements made here and more detailed descriptions can be found in standard texts like Hua [5] or Narkiewicz [12], [13].

Let $D<0$ be a squarefree integer and let $K=Q(\sqrt{D})$ be the quadratic field which is defined by adjoining $\sqrt{D}$ to the set of rational numbers $\mathcal{Q}$. If $\alpha \in K$ we denote by $\bar{\alpha}$ the complex conjugate of $\alpha$, by $\operatorname{Tr}(\alpha)$ the trace of $\alpha$, i.e. the value of $\alpha+\bar{\alpha}$, and by $N(\alpha)$ the norm of $\alpha$, i.e. the value of $\alpha \cdot \bar{\alpha}$. Note that $N(\alpha) \geq 0$ for every $\alpha \in K$.

We define

$$
r= \begin{cases}1 & \text { if } D \equiv 2,3 \bmod 4 \\ 2 & \text { if } D \equiv 1 \bmod 4\end{cases}
$$

Then the discriminant of $K$ is given by

$$
\Delta=\frac{4 D}{r^{2}}
$$

For $\alpha, \beta \in K$ put $[\alpha, \beta]=\alpha \mathscr{Z}+\beta \mathbb{Z}$. Moreover, let

$$
\omega=\frac{r-1+\sqrt{D}}{r}
$$

Then the ring of algebraic integers in $K$ is given by

$$
\mathcal{O}_{\mathcal{N}}=[1, \omega]
$$


The fractional ideals of ( $)$. form a multiplicative abelian group denoted by $F$. By 1 -ideal we denote the neutral element in this group. The principal ideals of $(\mathcal{H})$ form a subgroup of $F$ denoted by $P$. The factor group $F / P$ is called the class group of $C_{\lambda}$. clenoted by $C_{\Delta}$. The class group is finite, its orcler is called the class number of $\left(\nu_{\lambda}\right.$ and is denoted by $h$. The elements of the class group are called the ideal classes. Two ideals of $\mathcal{C}_{\lambda}$ : are said to be equivalent if they are in the same ideal class.

Every integral ideal a of ()$_{\lambda}$ can be written in the form

$$
\mathrm{a}=[a, b+c \omega]
$$

where $a, c \in \mathbb{Z}^{1}, b \in \mathbb{Z}$ and

$$
c|a, c| b, a c \mid N(b+c w) \text {. }
$$

For a given ideal a the integers $a$ and $c$ are uniquely determined and $b$ is unique modulo $a . a$ is the least positive rational integer in a, denoted by $L(\mathrm{a})$. If $c=1$ then a is called primitive.

Every integral primitive ideal a of $\mathcal{O}_{K}$ can be uniquely presented in the form

$$
\mathrm{a}=[L(\mathrm{a}), b+\omega]
$$

with $b \in \mathbb{Z}$ and

$$
-L(a)<\operatorname{Tr}(b+\omega) \leq L(a) .
$$

This is called the normal presentation of a.

An integral primitive ideal a whose normal presentation is a $=$ $[L(a), b+\omega]$ is called reduced if

$$
\begin{aligned}
|b+\omega| & \geq L(\mathrm{a}), \\
\operatorname{Tr}(b+\omega) & >0 \text { if }|b+\omega|=L(\mathrm{a}) .
\end{aligned}
$$

There are the following main facts concerning reduced ideals:

- For each reduced ideal we have $L(\mathrm{a})<\sqrt{|\Delta| / 3}$.

- Every ideal class of $\mathcal{O}_{\mathcal{K}}$ contains exactly one reduced ideal.

Each ideal class can be represented by its reduced ideal. So the arithmetic in the class group of imaginary quadratic fields can be reduced to ideal-arithmetic: In order to determine the product of two ideal classes multiply their reduced ideals and compute the reduced ideal equivalent to this product. 
Since every reduced ideal is presented by a pair of integers which are less than $\sqrt{|\Delta| / 3}$ in absolute value class groups can be used in the Diffie-Hellman scheme if multiplication and reduction of ideals can be carried out efficiently. The secret key must be one of the integers of the normal presentation of the reduced ideal computed in the scheme.

\section{THE ALGORITHMS}

In [2] it is pointed out that $D$ has to be of order of magnitude $10^{200}$ to guarantee high security of the scheme. Also the exponents should be of this order of magnitude. So first of all we need a multi-precision integer-arithmetic for implementing the scheme. For details see [6] or [1].

After the initialization all computations have the following structure: For an integer $n$ and an ideal class $\Gamma$ given by its reduced ideal $a_{\Gamma}$ compute the reduced ideal in $\Gamma^{n}$, i.e. the reduced ideal equivalent to $\left(a_{\Gamma}\right)^{n}$. This can be done by means of the well known fast exponentiation technique described in [6, p. 441].

\section{Algorithm 3.1 (Exponentiation of ideal classes)}

Input: Reduced ideal a, exponent $n \in \mathbb{Z}^{>0}$.

Output: Reduced ideal b equivalent to $\mathrm{a}^{n}$.

(1) $N \leftarrow n ; \mathrm{b} \leftarrow 1$-ideal; $\mathrm{c} \leftarrow \mathrm{a}$;

(2) if $N$ is even then goto (5);

(3) $\mathrm{b} \leftarrow \mathrm{c} \cdot \mathrm{b}$;

(4) reduce $b$;

(5) $N \leftarrow\lfloor N / 2\rfloor$;

(6) if $N=0$ terminate.

(7) $\mathrm{c} \leftarrow \mathrm{c} \cdot \mathrm{c}$;

(8) reduce $\mathrm{c}$ and goto (2);

For practical purposes it is more convenient to represent an integral primitive normally presented ideal $\mathrm{a}=[L(\mathrm{a}), b+\omega]$ by $(A, B) \in \mathbb{Z}^{2}$ with 
$A=r L(\mathrm{a})$ and $B=r b+r-1$. Note that the new presentation is well defined and unicque.

For the multiplication we use an algorithm based on Shanks [15] which computes a primitive ideal equivalent to the product of two primitive ideals:

\section{Algorithm 3.2 (Multiplication of ideals)}

Input: Two primitive ideals $\left(A_{1}, B_{1}\right),\left(A_{2}, B_{2}\right)$.

Output: Primitive ideal $\left(A_{3}, B_{3}\right)$ equivalent to the product of the input ideals.

(1) $G^{\prime}-\operatorname{gcd}\left(A_{1}, A_{2}\right)$;

compute the coefficient $V^{\prime}$ in $A_{1} V^{\prime}+A_{2} W^{\prime}=G^{\prime}$;

(2) $B_{3} \leftarrow V^{\prime} A_{1}\left(B_{2}-B_{1}\right)$;

(3) $A_{3} \leftarrow r A_{1} A_{2}$;

(4) if $G^{\prime}=1$ then goto (8);

(5) $G \leftarrow \operatorname{gcd}\left(G^{\prime},\left(B_{1}+B_{2}\right)\right)$;

compute the coefficients $U^{\prime}, U$ in $U^{\prime} G^{\prime}+U\left(B_{1}+B_{2}\right)=G$;

(6) $B_{3}-\left[B_{3} U^{\prime}+U\left(D-B_{1}^{2}\right)\right] / G$;

(7) $A_{3} \leftarrow A_{3} / G^{2}$

(8) $B_{3} \leftarrow B_{1}+B_{3}$;

If the ideals to be multiplied are equal then step 1 of the multiplication algorithm can be replaced by

$$
G=A_{1}, W^{\prime}=1, V^{\prime}=0
$$

because $A_{1}=A_{2}$. This simplification leads to the following algorithm for squaring an ideal:

Algorithm 3.3 (Squaring of an ideal)

Input: Primitive ideal $\left(A_{1}, B_{1}\right)$.

Output: Primitive ideal $\left(A_{3}, B_{3}\right)$ equivalent to the square of the input ideal.

(1) $G \leftarrow \operatorname{gcd}\left(A_{1}, 2 B_{1}\right)$;

compute the coefficient $U$ in $U^{\prime} A_{1}+2 U B_{1}=G$; 
(2) $B_{3}-B_{1}+\left[U\left(D-B_{1}^{2}\right)\right] / G$;

(3) $A_{3}-r\left(A_{1} / G^{\prime}\right)^{2}$

(4) terminate.

Now we discuss ideal reduction. In order to decrease the size of the coefficients of the ideal representation whenever it is possible the ideals are reduced after each multiplication in Algorithm 3.1. So the reduction algorithm is applied very frequently and therefore it is necessary to have a fast reduction method. Our method is a refinement of the following well known algorithm (see [8] or [9]).

Algorithm 3.4 (Reduction of ideals, classical version) Input: Primitive ideal $\left(A^{\prime}, B^{\prime}\right)$.

Output: Reduced ideal $(A, B)$ (in normal presentation) equivalent to the input ideal.

(1) $A \leftarrow A^{\prime} ; B \leftarrow B^{\prime}$;

(2) $B \leftarrow \operatorname{round}(B / A) \cdot A-B$;

(3) $A_{N} \leftarrow\left(B^{2}-D\right) / A$;

(4) if $A_{N}<A$ then $A \leftarrow A_{N}$ and goto (2);

(5) if $(2 B<A)$ and $\left(B<0\right.$ or $\left.A<A_{N}\right)$ then $B \leftarrow-B$;

In step 2 of the algorithms "round" means a multi precision integer function which computes the rounded quotient of two integers. This can be done by the following algorithm:

Algorithm 3.5 (Rounded quotient of two integers)

Input: Integers $A$ and $B, B \neq 0$.

Output: Integer $C$ with $C=\operatorname{round}(A / B)$.

(1) $C \leftarrow A / B ; R \leftarrow A \bmod B$;

(2) if $2|R| \geq|B|$ then [if $A>0$ then $C \leftarrow C+1$ else $C \leftarrow C-1$ ];

Now we present the refinement of the algorithm which was theoretically described in [2]. 
Algorithm 3.6 (Reduction of ideals, optimized version) Input: Primitive ideal $\left(A^{\prime}, B^{\prime}\right)$.

Output: Reduced ideal $(A, B)$ (in normal presentation) equinalent to the input ideal.

(1) $A-A^{\prime}$

if $B^{\prime}<0$ then $s-1$ else $s-1$;

$B-\left|B^{\prime}\right|$;

(2) $Q-B / A: R-B \bmod A ; M-A-2 R$ :

if $M \geq 0$ then $B-R$ and $s--s$ else $B-R+M$;

$A_{N}-\left(B^{2}-D\right) / A$;

(3) if $A_{N}<A$ then $A_{O}-A$ and $A-A_{N}$ else goto (6);

(4) $Q \leftarrow B / A ; R \leftarrow B \bmod A ; M \leftarrow A-2 R$;

(5) $A_{N} \leftarrow A_{O}-(R+B) \cdot Q$;

If $M \geq 0$ then $B \leftarrow R$ and $s \leftarrow-s$ else $B \leftarrow R+M$ and

$A_{N} \leftarrow A_{N}+M$;

goto (3);

(6) if $s<0$ then $B \leftarrow-B$;

if $(2 B<A)$ and $\left(B<0\right.$ or $\left.A<A_{N}\right)$ then $B \leftarrow-B$;

Comparing the two versions of the algorithm we see that the number of iterations in both algorithms and also the sequences of the values for $A$ and $B$ computed in both algorithms are equal. There are two main differences between the two versions:

The computation of the new value of $A$ in each iteration in the optimized version needs one division of multi precision integers less than in the first version because the division in step 3 is avoided. Instead of this the value of $A$ from the preceeding iteration is used. Therefore this simplification cannot be made up in the first iteration. So step 2 of Algorithm 3.6 contains the first iteration of Algorithm 3.4.

The computation of the rounded quotient of $A$ and $B$ is avoided in the optimized version. Moreover the sign of $B$ is stored in a seperate variable $s$. In this way one multiplication of multi precision integers in each iteration is avoided.

The following theorem makes sure that algorithm 3.6 indeed computes the reduced ideal equivalent to the input ideal in finitly many 
iterations. Moreover an estimation for the number of iterations is given. In [2] this theorem was proved for Algorithm 3.4 which requires one more iteration.

Theorem 3.1 Let $(A, B)$ represent a primitice ideal. Algorithm 3.4 finds the reduced ideal equivalent to $(A, B)$ in at most i iterations of the steps $3: 4$ and 5 where

$$
i=\max \left\{0,\left\lfloor\frac{1}{2} \log \left(\frac{3 A}{5 \sqrt{|D|}}\right)\right\rfloor+1\right\} .
$$

Finally we describe the initialization where we have to find an appropriate starting ideal class for a given value of $D$. We chose - at random - a prime number $q(q \equiv 3 \bmod 4)$ such that $D$ is quadratic residue modulo $q$. Then

$$
\begin{aligned}
& A=r \cdot q \\
& B=D^{\frac{q+1}{4}} \bmod r q
\end{aligned}
$$

satisfy the condition

$$
r A \mid B^{2}-D
$$

So $(A, B)$ represents a primitive ideal. Reduction of this ideal gives the starting ideal class.

\section{THE COMPLEXITY}

First we compute the size of the integers occuring in the computations of our key exchange system for a fixed value of $D$.

\section{Theorem 4.1}

(a) If $(A, B)$ is a reduced ideal in normal presentation then

$$
\begin{aligned}
A & \leq 2 \sqrt{\frac{|D|}{3}} \\
|B| & \leq \sqrt{\frac{|D|}{3}}
\end{aligned}
$$


(b) If $(A, B)$ is the primitive ideal computed by Algorithm 3.2 or 3.3 then

$$
\begin{aligned}
A & \leq \frac{8|D|}{3} \\
|B| & \leq \frac{16|D|}{3} \sqrt{\frac{|D|}{3}}
\end{aligned}
$$

provided that the input ideals were reduced and given in nomal presentation.

(c) The size of the integers occuring in the computations of our key exchange system is bounded by

$$
M=3|D| \sqrt{|D|} .
$$

Proof:

(a) Let $\mathrm{a}=[L(\mathrm{a}), b+\omega]=(A, B)$. Then, according to the definition of $A$ and $B$ in section 4 , we have $A=r L(a)$ and $B=r b+r-1$. Moreover

$$
b+\omega=\frac{B+1}{r}-1+\frac{r-1+\sqrt{D}}{r}=\frac{B+\sqrt{D}}{r}
$$

and therefore

$$
\operatorname{Tr}(b+\omega)=\frac{2 B}{r} \leq 2 B .
$$

Since $\mathbf{a}$ is reduced we have

$$
A=r L(\mathrm{a})<r \sqrt{\frac{|\Delta|}{3}}=r \sqrt{\frac{4|D|}{3 r^{2}}}=2 \sqrt{\frac{|D|}{3}} .
$$

Since $\mathbf{a}$ is in normal presentation we have $|\operatorname{Tr}(b+\omega)| \leq I(\mathbf{a})$ and therefore

$$
|B| \leq \frac{L(\mathrm{a})}{2}=\sqrt{\frac{|D|}{3}} .
$$

(b) In Algorithm 3.2 the new value for $A$ is computed in steps 3 and 7. From these steps and part (a) of this theorem we get

$$
A \leq 2 \cdot\left(2 \sqrt{\frac{|D|}{3}}\right)^{2}=\frac{8|D|}{3} .
$$

The new value for $B$ is computed in steps 2,4 and 8 . The coefficients $V^{\prime}, V, U^{\prime}$ in step 2 and 5 can be choosen less than $2 \sqrt{\frac{|D|}{3}}$. So

$$
B \leq 2 \sqrt{\frac{|D|}{3}} \cdot 2 \sqrt{\frac{|D|}{3}} \cdot \sqrt{\frac{|D|}{3}} .
$$


The same arguments apply to Algorithm 3.3.

(c) The input ideals for the multiplication and squaring algorithm are always reduced ideals in normal presentation. The input ideals for the recluction algorithm are always results of multiplication and scuuaring. Moreover the successive values of $A$ in the reduction algorithm are strictly decreasing with the exeption of the last one and therefore the size of the input value of $A$ is an upper bound for all values of $A$ and $B$ occuring in the algorithm. So by (a) and (b) we see the maximum integer occuring in the system is bounded by

$$
\frac{16|D|}{3} \sqrt{\frac{|D|}{3}}=\frac{16 \sqrt{3}}{9}|D| \sqrt{|D|}<3|D| \sqrt{|D|} .
$$

Note that by part (c) of this theorem we also can estimate the number of computer words necessary to store the integers used in the algorithm. If for example $D$ has 200 decinal digits then our multi precision arithmetic package must be able to handle numbers of 301 decimal digits.

Next we list the number of elementary operations (additions, multiplications and divisions) with multi precision integers for each of our algorithms. Multiplication by $r$ is considered as an addition because $r$ is either 1 or 2 .

To analyze the multiplication and squaring algorithm we first need to know the number of elementary operations necessary to compute the gcd's. This computation can be done by the euclidian algorithm [6, p. 325]. If one has to compute a ged and both coefficients of its representation the euclidian algorithm needs one division, three additions and three multiplications in each division step. This case will be called "gcd2". If one has to compute a gcd and only one of the coefficients of its representation one can avoid some computations. Then the euclidian algorithm only needs one division, two additions and two multiplications in each division step. This case will be called "ged1".

\section{Theorem 4.2}

(a) The maximum number of division steps in the euclidian algorithm (see [6, p. 325]) used for the computation of the god's in the multiplication and squaring algorithm is

$$
M^{\prime} \leq \frac{3}{4} \log |D|+1 .
$$


(b) In case $G^{\prime}=1$ Algorithm 3.2 requires at most $\left(3+2 M^{\prime}\right)$ additions: $\left(3+2 M I^{\prime}\right)$ multiplications and $M^{\prime}$ dicisions of multi precision integers.

(c) In case $C^{\prime \prime}>1$ Algorithm 3.2 requires at most $\left(6+5 \mathrm{M}^{\prime}\right)$ additions: $\left(T+5 M^{\prime}\right)$ multiplications and $\left(2+2 M^{\prime}\right)$ divisions of multi precision integers.

(d) Algorithm 3.3 requires at most $\left(3+2 M^{\prime}\right)$ additions, $\left(3+2 M^{\prime}\right)$ multiplications and $\left(2+M^{\prime}\right)$ divisions of multi precision integers.

Proof:

(a) From the bounds given in Theorem 4.1 (a) for the input values of the multiplication and squaring algorithm and by the listings of these algorithms in the previous section we see that the input values for the gcd's are bounded by $2 \sqrt{\frac{|D|}{3}}$. By Corollary $L$ in $[6, p .343]$ we can conclude that the number of division steps required when the euclidian algorithm is applied to numbers $u$ and $v$ with $0 \leq u, v<N$ is at most $4.8 \log _{10} N+0.68$. So in our special case we have

$$
\begin{aligned}
M^{\prime} & \leq 4.8 \log _{10}\left(2 \sqrt{\frac{|D|}{3}}\right)+0.68 \\
& =4.8 \log _{10} \frac{2}{\sqrt{3}}+\frac{4.8}{\log 10} \log \sqrt{|D|}+0.68 \\
& \leq \frac{3}{2} \log \sqrt{|D|}+1 \\
& =\frac{3}{4} \log |D|+1 .
\end{aligned}
$$

(b) For $G^{\prime}=1$ Algorithm 3.2 requires three additions, three multiplications and one gcdl.

(c) For $G^{\prime}>1$ Algorithm 3.2 requires three additions, seven multiplications, two divisions, one gcdl and one gcd2.

(d) Algorithm 3.3 requires three additions, three multiplications, two divisions and one gcdl.

Now we turn to the reduction algorithm. Here we first have to compute the maximum number of iterations. 
Theorem 4.3

(a) The maximum number of iterations in the optimized lersion of the reduction algorithm is

$$
I^{\prime \prime} \leq \frac{1}{4} \log |D|+2 .
$$

(b) Algorithm 3.4 requires $\left(1+4\left(M^{\prime \prime}+1\right)\right)$ additions, $2\left(M^{\prime \prime}+1\right)$ multiplications and $2\left(M^{\prime \prime}+1\right)$ divisions of multi precision integers. Here the operations caused by the seperate "round"-algorithm are included.

(c) Algorithm 3.6 requires $\left(5+6 M^{\prime \prime}\right)$ additions, $\left(1+M^{\prime \prime}\right)$ multiplications and $\left(2+M^{\prime \prime}\right)$ divisions of multi precision integers.

Proof:

(a) Using the bound given in Theorem 4.1 (b) we have

$$
A \leq \frac{8|D|}{3}
$$

for the input ideal of the reduction algorithm. From Theorem 3.1 we conclude that the number of iterations in Algorithm 3.6 is bounded by

$$
\begin{aligned}
M^{\prime \prime} & \leq \frac{1}{2} \log \left(\frac{3 \frac{8|D|}{5}}{5 \sqrt{|D|}}\right)+1 \\
& =\frac{1}{2} \log \left(\frac{8}{5} \sqrt{|D|}\right)+1 \\
& =\frac{1}{2} \log \frac{8}{5}+\frac{1}{4} \log |D|+1 \\
& \leq \frac{1}{4} \log |D|+2 .
\end{aligned}
$$

(b) The number of iterations in Algorithm 3.4 is one more than in Algorithm 3.6. Each iteration (steps 2, 3 and 4) requires two additions, two multiplications, one division and one call of the round procedure in which there are two additions and one division. Step 5 of the reduction algorithm requires one additional addition.

(c) Each iteration (steps 3,4 and 5) in Algorithm 3.6 requires six additions, one multiplication and one division. Additionally, steps 2 and 6 require five additions, one multiplication and two divisions.

Now we have to examine Algorithm 3.1 for the exponentiation of ideal classes. 
Theorem 4.4 If the exponents a and $b$ are bounded by $a \cdot b<\sqrt{|D|}$ then the total number of iterations performed in all calls of the exponentiation algorithm in the key exchange system is at most

$$
M^{\prime \prime \prime} \leq \log |D|+4
$$

Proof: The number of iterations in algorithm 3.1 for an arbitrary exponent $n$ is lower than $\lfloor\log n\rfloor+1$ (see $[6,443]$ ). Each exponent is used twice in the scheme. Therefore the total number of iterations performed in the four calls of the exponentiation algorithm is bounded by

$$
\begin{aligned}
M^{\prime \prime \prime} & \leq 2(\log a+1+\log b+1) \\
& \leq 2 \log (a \cdot b)+4 \\
& \leq 2 \log \sqrt{|D|}+4 \\
& =\log |D|+4
\end{aligned}
$$

Finally we give an upper bound for the number of elementary operations performed in the key exchange system which follows from Theorems $4.2,4.3$ and 4.4 .

Theorem 4.5 Using the optimized version of the reduction algorithm our key exchange system takes at most

$$
\begin{array}{ll}
217+\frac{169}{2} \log |D|+\frac{33}{4} \log ^{2}|D| & \text { additions, } \\
95+\frac{185}{4} \log |D|+\frac{23}{4} \log ^{2}|D| & \text { multiplications, } \\
64+\frac{105}{4} \log |D|+\frac{11}{4} \log ^{2}|D| & \text { divisions }
\end{array}
$$

of multi-precicison integers.

Now we give the asymptotical bit complexity for our algorithms and for the whole method. Clearly the complexity depends on the implementation of the multi precision routines. Using the so called "Classical Algorithms" (see [6, p. $250 \mathrm{ff}$.$] ) multiplication and divsion of n$-bit numbers require $O\left(n^{2}\right)$ bit operations. Using the so-called "Fast Multiplication Techniques" these operations numbers require only $O(n \log n \log \log n)$ bit-operations (see [1, p. 272, 286]). 
(a) The algorithms for squaring and multiplication of ideals and both versions of the reduction algorithm have bit complexity

(i) $O\left(\log ^{3}|D|\right)$ if the classical algorithms are used,

(ii) $O\left(\log ^{2}|D| \log \log |D| \log \log \log |D|\right)$ if fast multiplication technique is used.

(b) The method for public key exchange has bit complearity

(i) $O\left(\log ^{4}|D|\right)$ if the classical algorithms are used,

(ii) $O\left(\log ^{3}|D| \log \log |D| \log \log \log |D|\right)$ if fast multiplication technique is used.

Proof: Both statements follow immediately from the previous results. Note that according to Theorem 4.1 (c) the binary length of the integers occuring in our scheme is $O(\log |D|)$.

The theorem shows that both versions of the reduction algorithm have the same asymptotical complexity. The improvement in the optimized version only affects the $O$-constant as can be seen from Theorem 4.3 .

We see that using fast multiplication techniques the running time of our method for public key exchange is a cubic polynomial in the length of the input data. Therefore it is executable for big exponents and big discriminants.

\section{RUNNING TIME STATISTICS}

The method for public key exchange has been implemented on a SIEMENS 7580-S computer of the University of Düsseldorf [4]. The programming language was FORTRAN-77. For the multi-precision arithmetic we used the classical algorithms. These and some procedures to get the running time statistics presented below were taken from the number theoretic subroutine library KANT.

We computed many examples where the value of $D$ was the product of two prime numbers each of size up to $10^{100}$. This choice of $D$ was done according to the remarks in [2] concerning the security of the method. Both cases $r=1$ and $r=2$ occured equally often. 
In the table below we present the statistics of 32 of these examples. Here the size of $D$ varies between $10^{120}$ and $10^{200}$. For each size of $D$ we took several exponents of different size. The columns of the table have the following contents:

(1) Number of the example

(2) Size of $D$ (number of decimals)

(3) Size of the product of the exponents (number of decimals)

(4) Number of calls of the squaring algorithm

(5) Number of calls of the nultiplication algorithm

(6) Number of calls of the reduction algorithm

(7) Average number of iterations in the reduction algorithm

(8) Maximum number of iterations in the reduction algorithm

(9) Theoretical bound for the number of iterations in the reduction algorithm

(10) Average running time of the squaring algorithm

(11) Average running time of the multiplication algorithm

(12) Average running time of the classical version of the reduction algorithm

(13) Average running time of the optimized version of the reduction algorithm

(14) Total running time of the program with the optimized version of the reduction algorithm

All times are given in seconds (CPU).

From the structure of the exponentiation algorithm we know the connection between the exponents and the number of calls of our algorithms: The number of squarings (col. 4) is exactly twice the sum of the logarithms of the two exponents. The number of multiplications (col. 5) is twice the number of One's in the binary representation of the exponents. The number of reductions (col. 6) minus 1 is exactly the sum of 
the colunns 4 and 5 because there is a reduction after each scluaring or multiplication and one reduction in the initialization.

The theoretical bound for the number of iterations in the reduction algorithm is computed from the formula in Theorem 4.3 (a) with a value of $D$ rounded to the next power of ten.

\section{Table (Running time statistics)}

\begin{tabular}{|c|c|c|c|c|c|c|c|c|c|c|c|c|c|}
\hline & \multicolumn{2}{|c|}{ Decis } & \multirow{2}{*}{\multicolumn{3}{|c|}{$\begin{array}{c}\text { Subroutine } \\
\text { Calls }\end{array}$}} & \multirow{2}{*}{\multicolumn{3}{|c|}{$\begin{array}{l}\text { Iterations } \\
\text { in reduction }\end{array}$}} & \multicolumn{4}{|c|}{ Average running time } & \multirow{3}{*}{$\begin{array}{l}\text { Total } \\
\text { running } \\
\text { time }\end{array}$} \\
\hline & & & & & & & & & & & Red & tion & \\
\hline & $D$ & $a \cdot b$ & Squ & Mul & Red & aver & $\max$ & theo & Squ & Mul & Cla & Opt & \\
\hline (1) & (2) & (3) & (4) & (5) & (b) & (7) & (8) & (9) & (10) & (11) & (12) & (13) & (14) \\
\hline 1 & 198 & 20 & 62 & 30 & 93 & 57 & 80 & 160 & 0.23 & 0.23 & 1.60 & 0.27 & 47.11 \\
\hline 2 & 198 & 30 & 192 & 88 & 281 & 64 & 79 & 166 & 0.25 & 0.28 & 1.80 & 0.30 & 159.83 \\
\hline 3 & 197 & 50 & 324 & 162 & 487 & 65 & 77 & 165 & 0.27 & 0.28 & 1.81 & 0.30 & 280.79 \\
\hline 4 & 197 & 71 & 468 & 236 & 705 & 66 & 82 & 165 & 0.27 & 0.28 & 1.82 & 0.30 & 408.24 \\
\hline 5 & 197 & 97 & 640 & 294 & 935 & 66 & 80 & 165 & 0.27 & 0.27 & 1.84 & 0.30 & 540.30 \\
\hline 6 & 182 & 11 & 66 & 32 & 99 & 51 & 71 & 153 & 0.20 & 0.21 & 1.25 & 0.22 & 42.54 \\
\hline 7 & 182 & 30 & 190 & 82 & 273 & 59 & 79 & 153 & 0.23 & 0.24 & 1.44 & 0.26 & 133.59 \\
\hline 8 & 182 & 48 & 310 & 140 & 451 & 80 & 75 & 153 & 0.23 & 0.24 & 1.48 & 0.27 & 225.44 \\
\hline 9 & 182 & 71 & 466 & 238 & 705 & 61 & 74 & 153 & 0.24 & 0.24 & 1.50 & 0.27 & 356.13 \\
\hline 10 & 182 & 93 & 608 & 286 & 895 & 62 & 74 & 153 & 0.24 & 0.24 & 1.51 & 0.27 & 454.34 \\
\hline 11 & 178 & 19 & 118 & 62 & 181 & 54 & 72 & 148 & 0.21 & 0.21 & 1.28 & 0.23 & 80.75 \\
\hline 12 & 177 & 40 & 246 & 132 & 379 & 57 & 7.4 & 148 & 0.22 & 0.22 & 1.34 & 0.25 & 177.06 \\
\hline 13 & 177 & 60 & 394 & 202 & 597 & 59 & 75 & 148 & 0.23 & 0.23 & 1.39 & 0.25 & 288.64 \\
\hline 14 & 177 & 79 & 518 & 238 & 757 & 59 & 75 & 148 & 0.23 & 0.23 & 1.40 & 0.25 & 363.59 \\
\hline 15 & 167 & 10 & 58 & 26 & 85 & 48 & 72 & 140 & 0.18 & 0.18 & 1.03 & 0.20 & 32.25 \\
\hline 16 & 167 & 40 & 256 & 122 & 379 & 55 & 69 & 140 & 0.20 & 0.21 & 1.18 & 0.22 & 162.92 \\
\hline 17 & 166 & 60 & 394 & 202 & 597 & 55 & 70 & 139 & 0.20 & 0.20 & 1.20 & 0.22 & 255.87 \\
\hline 18 & 166 & 79 & 518 & 238 & 757 & 55 & 70 & 139 & 0.20 & 0.21 & 1.22 & 0.23 & $326: 39$ \\
\hline 19 & 157 & 10 & 62 & 40 & 103 & 46 & 64 & 132 & 0.17 & 0.17 & 0.89 & 0.18 & 46.50 \\
\hline 20 & 157 & 30 & 196 & 98 & 295 & 51 & 66 & 132 & 0.18 & 0.19 & 0.99 & 0.20 & 113.45 \\
\hline 21 & 152 & 51 & 334 & 182 & 517 & 50 & 63 & 128 & 0.18 & 0.18 & 0.94 & 0.19 & 193.32 \\
\hline 22 & 158 & 71 & 466 & 228 & 695 & 53 & 67 & 133 & 0.19 & 0.19 & 1.04 & 0.21 & 276.74 \\
\hline 23 & 146 & 10 & 62 & 30 & 93 & 42 & 59 & 123 & 0.14 & 0.16 & 0.72 & 0.16 & 28.40 \\
\hline 24 & 147 & 30 & 190 & 84 & 275 & 47 & 60 & 124 & 0.16 & 0.16 & 0.83 & 0.18 & 93.22 \\
\hline 25 & 136 & 50 & 328 & 188 & 517 & 46 & 59 & 115 & 0.15 & 0.15 & 0.72 & 0.16 & 161.26 \\
\hline 26 & 142 & 71 & 466 & 238 & 705 & 48 & 62 & 119 & 0.16 & 0.17 & 0.81 & 0.18 & 240.87 \\
\hline 27 & 127 & 20 & 126 & 44 & 171 & 40 & 51 & 107 & 0.13 & 0.13 & 0.58 & 0.14 & 45.33 \\
\hline 28 & 133 & 39 & 246 & 132 & 379 & 44 & 56 & 112 & 0.14 & 0.14 & 0.67 & 0.15 & 111.96 \\
\hline 29 & 127 & 60 & 394 & 202 & 597 & 43 & 53 & 107 & 0.13 & 0.14 & 0.61 & 0.14 & 167.23 \\
\hline 30 & 123 & 20 & 128 & 54 & 183 & 40 & 54 & 104 & 0.12 & 0.13 & 0.54 & 0.13 & 47.35 \\
\hline 31 & 129 & 41 & 258 & 140 & 399 & 43 & 55 & 109 & 0.13 & 0.14 & 0.62 & 0.15 & 113.18 \\
\hline 32 & 124 & 59 & 380 & 176 & 557 & 42 & 52 & 104 & 0.13 & 0.13 & 0.58 & 0.14 & 149.86 \\
\hline
\end{tabular}


The comparison of the values in column 8 and 9 shows that the maximum number of iterations in the reduction algorithms is about half of the theoretical bound.

The comparision of the average ruming time for the two versions of the reduction algorithm shows that there is indeed an important speeding up. The optimized version only requires $20 \%$ or less of the running time of the classical version. Most of this gain is caused by avoiding one multi precision division in each iteration. Note that the optimized version of the reduction algorithm is nearly as fast as the multiplication of ideals.

Note also that there is almost no difference in the average running time of the multiplication and the squaring algorithm. Here the theoretical improvement has no practical effect.

The examination of the total running time of the program confirms the complexity result given in Theorem 4.6.

Now we give the data computed by our program for the first example mentioned in the table above. This example has the highest values of D.

We list the information to be transmitted over the public channel $\left(D\right.$, the starting ideal class $\Lambda$ and the ideal classes $\Phi=\Lambda^{a}$ and $\left.\Psi=\Lambda^{b}\right)$ and also the secret information (the exponents $a$ and $b$ and the ideal class $\Gamma=\Lambda^{a b}$ which gives the secret key).

Note that even if we use low exponents like in the first example the secret key has a reasonable size of about $\sqrt{|D|}$. Nevertheless one should choose larger exponents like in the fifth example to have greater security.

\section{Example}

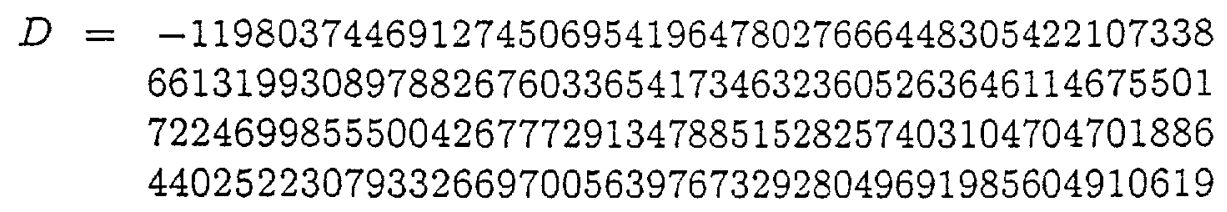

Secret exponents:

$$
\begin{array}{lr}
\text { Secret exponents: } \quad a=543210, & b=7980 \\
\text { Starting ideal class } \Lambda: & A=982, \quad B=31
\end{array}
$$

Ideal class $\Phi=\Lambda^{a}$ : 
$A=971429613036373986082445759917205522588066014949$ 1.3086285698000466252477914793685446384554925704750

$B=395993438464946485994639174385199545445240652683$ $753364678843011371468419191822333+72651118628470641$

Ideal class $\Psi=\Lambda^{b}$ :

$A=3245184228591470294850077585641939122326353850768$ $802416 \div 263208946226417449854469021281560392496.5610$

$B=1346467765516260169857822840300447086991473982874$ 11630817948537340265799828623744461455733888148671

Ideal class $\Gamma=\Lambda^{a b}$ :

$A=3326434363518178161689675328487011083251016168417$ 44606450020063171247803446369585670411999866011434

$B=-97577809448926539679909701428640738988823144173$ 53203195510945029218250620415723441254072993437105

\section{References}

[1] A.V. Aho, J.E. Hopcroft, J.D. Ullman, The design and analysis of computer algorithms, Addison-Wesley, Reading, Massachusetts, 1974.

[2] J. Buchmann and H.C. Williams, A key exchange system based on imaginary quadratic fields, Journal of Cryptology 1 (1989), to appear.

[3] W. Diffie and M. Hellman, New directions in cryptography, IEEE Transactions on Information theory 22 (1976), $472-492$.

[4] S. Düllmann, Ein neues Verfahren zum öfentlichen Schlüsselaustausch, Staatsexamensarbeit, Universität Düsseldorf, 1988.

[5] Hua Loo Keng, Introduction to number theory, Springer Verlag, Berlin and New York, 1982.

[6] D.E. Knuth, The art of computer programming, vol. 2: Seminumerical algorithms, Addison-Wesley, Reading, Massachusetts, 2. Aullage, 1981. 
[7] N. Koblitz, A Family of Jacobians Suitable for Discrete Log Cryptosystems, to appear in: Proceedings of Crypto'88, Lecture Notes of Computer Science, Springer-Verlag.

[8] J.C. Lagarias, Worst-Case Complexity Bounds for Algorithms in the Theory of Integral Quadratic Forms, Journal of Algorithms 1 (1980), $142-186$.

[9] H.W Lenstra, Jr., On the calculation of regulators and class numbers of quadratic fields, London Math. Soc. Lecture Notes 56 (1982), $123-150$.

[10] K.S. McCurley, A Key Distribution System equivalent to Factoring, preprint, 1987.

[11] V. Miller, Use of Elliptic Curves in Cryptography, Advances in cryptology (Proceedings of Crypto'85), Lecture Notes in Computer Science 218 (1986), Springer-Verlag, NY, 417 - 426.

[12] W. Narkiewicz, Elementary and analytic theory of algebraic numbers, Warszawa, 1974.

[13] W. Narkiewicz, Number theory, Warszawa, 1977, engl. edition 1983.

[14] R.W.I. Odoni, V. Varadharajan and P.W. Sanders, Public Key Distribution in Matrix Rings, Electronic Letters 20 (1984), 386 387.

[15] D. Shanks, Class number, a theory of factorization and genera, Proc. Symposia in Pure Mathematics 20 (1971), 415 - 440. 